\title{
Enseñando y aprendiendo el principio de conservación de la masa: interrogantres y desafíos
}

\author{
Héctor Odetti,* Claudia B. Falicoff,* \\ Pablo D. Húmpola,* Eduardo J. Bottani**
}

\section{Resumen}

Enseñar el principio de conservación de la masa presenta un problema para los estudiantes quienes no pueden emplear el mismo para explicar una situación física cotidiana. El origen del problema pareciera estar en la forma en la que el principio es enunciado y en conceptos adicionales ya enseñados. Los conceptos necesarios para comprender el principio de conservación de la masa están relacionados con la definición de sistema y sus diferentes tipos, los que son corrientemente empleados en los laboratorios de química. Se empleó un breve cuestionario para demostrar esta hipótesis. Aunque la muestra es reducida y no admite un análisis estadístico es posible confirmar el origen del problema. Como una solución se propone que los sistemas podrían ser estudiados previa o simultáneamente con el principio de conservación de la masa.

* Departamento de Química General e Inorgánica

Facultad de Bioquímica y Ciencias Biológicas UNL.

Ciudad Universitaria. Paraje El Pozo. CC 242 (3000) Santa Fe

Te: 54 - 342 - 4575212

e-mail: hodetti@fbcb.unl.edu.ar

** Instituto de Investigaciones Fisicoquímicas Teóricas y Aplicadas (INIFTA-UNLP-CIC-

CONICET). CC 16 Suc. 4 (1900) La Plata.FAX: 54-221-425-4642

e-mail: ebottani@inifta.unlp.edu.ar 